\title{
Efeitos de variáveis psicológicas na reatividade cardiovascular em momentos de stress emocional
}

\author{
Psychological effects on the cardiovascular \\ reactivity of stressful moments
}

\author{
Marilda Emmanuel Novaes LIPP' \\ Adriana FRARE' \\ Flavia Urbino dos SANTOS
}

\begin{abstract}
Resumo
A influência de características psicológicas no aumento da pressão arterial tem sido objeto de muitas indagações. Este trabalho analisou como, em momentos de stress, a reatividade cardiovascular é afetada por fatores psicológicos. Oitenta hipertensos responderam ao Inventario de Sintomas de Stress de Lipp, à Escala de Alexitimia de Toronto e ao Questionário de Assertividade, e passaram por uma sessão experimental envolvendo interações sociais estressantes. A pressão arterial e a freqüência cardíaca foram aferidas continuamente pelo monitor de pressão arterial Finapress. A pessoa era instruída em um momento a controlar suas emoções e em outro a expressá-las. Verificou-se uma correlação significativa entre nível de alexitimia e de inassertividade e reatividade da pressão arterial. Quando solicitados a expressarem emoções, a pressão arterial diastólica de alexitímicos e inassertivos sofreu aumentos significativos. A pressão arterial sistólica de pessoas assertivas e não alexitímicas sofreu aumentos significativos quando instruídas a inibirem as emoções.
\end{abstract}

Unitermos: alexitimia; expressar emoções; reatividade cardiovascular; stress.

\begin{abstract}
The effects of psychological traits on blood pressure increases have received much attention. This work aimed to verify if during periods of interpersonal stress the cardiovascular reactivity of hypertensives varies in accordance with psychological traits. Eighty mild-hypertensive adult subjects answered the Lipp Stress Symptom Inventory, the Toronto Alexithymia Scale and an Assertiveness Questionnaire, and participated in a conflictive role-play experimental session. Blood pressure and heart rate were recorded continuously via the Finapres methodology. Two types of instructions were given. At one time the subject was instructed to with hold emotions and at another time, the instruction given was to express emotions freely during the session. A significant correlation was found between alexithymia/inassertiveness and both diastolic and systolic blood pressures. When alexithymic/inassertive subjects were asked to express their feelings, the diastolic blood pressure increased significantly. Furthermore, when assertive and non-alexithymic participants were required to suppress their emotions the systolic blood pressure suffered significant increases.
\end{abstract}

Uniterms: alexithymia; expressed emotion; cardiovascular reactivity; stress.

$\cos$

' Pontifícia Universidade Católica de Campinas, Centro de Ciências da Vida, Laboratório de Estudos Psicofisiológicos do Stress. Av. John Boyd Dunlop, s/n., Ambulatório de Especialidades, Jd. Ipaussurama, 13060-904, Campinas, SP, Brasil. Correspondência para/Correspondence to: M.E.N. LIPP. E-mail: <mlipp@estresse.com.br>.

Agradecimento: Este trabalho contou com o financiamento do CNPq, Processo 550588/2002-7. As autoras agradecem ao Dr. Armando Miguel, cardiologista, pelo resultado cardiológico necessário ao estudo. 
A reatividade cardiovascular, ou seja, mudanças de pressão arterial ou freqüência cardíaca que ocorrem em resposta a uma situação ou evento específico, tem sido considerada uma função significativa na neurogenética das doenças coronarianas (Lovallo \& Gerin, 2003), da aterosclerose (Schwartz et al., 2003) e das neoplasias (Loures, Sant'Anna, Baldotto, Sousa \& Nóbrega, 2002). Algumas publicações (Light, Sherwood \& Turner, 1992; Treiber et al., 2003) têm discutido a hipótese da reatividade excessiva estar envolvida na patofisiologia da hipertensão arterial essencial, embora autores como Light et al. (1992) sugerem que a alta reatividade cardiovascular somente tem valor prognóstico para o desenvolvimento posterior da hipertensão arterial se além de uma história familiar de hipertensão, o stress estiver presente em altos níveis no decorrer da vida. Observando os cuidados necessários para evitar conclusões atributivas prematuras, estudos da reatividade cardiovascular têm sido realizados em laboratórios sob condição de controle experimental para averiguar o mecanismo de ação de aumentos pressóricos desencadeados pelo stress principalmente como resposta a estressores psicossociais (Fritz, Nagurney \& Helgeson, 2003; Kamarck \& Lovallo, 2003). Estudos brasileiros sobre reatividade cardiovascular mostram que o hipertenso exibe aumentos de pressão significativos quando submetido a sessões experimentais de stress emocional (Lipp, 2005; Lipp, Justo \& Melo Gomes, 2006).

Poderia se questionar se as mudanças em pressão verificadas em estudos laboratoriais são realmente o produto de uma reação ao stress ou variações normais de pressão arterial. Um estudo desenvolvido na Inglaterra com 1.259 homens mostrou que as variações em pressão verificadas em hipertensos durante sessões experimentais de stress psicológico não são devidas às oscilações regulares de pressão (Sheffield, Smith, Carrol, Shipley \& Marmot, 1997). O estudo utilizou medidas estatísticas com procedimentos compensatórios que permitiram concluir que embora oscilações normais existam, elas não podem justificar toda a reatividade cardiovascular verificada em inúmeros estudos.

O stress emocional seria, então, o principal elemento desencadeador da reatividade observada. Muitos autores têm dedicado seus esforços em busca de variáveis psicológicas que possam estar relacionadas

162 à produção de stress e têm pesquisado o papel das emoções na saúde, na vulnerabilidade ao stress e na reatividade cardiovascular.

Spielberger e Moscoso (1996) estudaram a associação entre stress e emoção e sua relação com os construtos psicológicos de ansiedade e raiva. Uma outra linha de pesquisa (Sandin, Chorot, Santed \& Jimenez, 1996) estabeleceu a relação entre sintomas físicos com alexitimia e outras variáveis psicológicas durante períodos de stress e verificaram a existência de uma relação entre alexitimia, estado de ansiedade, depressão, raiva e stress da vida diária, enquanto em outro trabalho os mesmos autores (Sandin, Santed, Chorot \& Valiente, 1996) estudaram a relação entre alexitimia, reatividade ao stress e doenças somáticas.

Alexitimia, definida por Sifneos (1972) como uma dificuldade da pessoa de identificar e de expressar vários tipos de emoções, têm sido identificada clinicamente em muitos pacientes hipertensos. Inassertividade e tendência à agressividade também são características que têm sido identificadas nessa população. Lipp e Rocha (1994) e Lipp (2005) verificaram que a inassertividade, definida por Wolpe (1973) como a inabilidade não só de defender os direitos pessoais e expressar opinião, mas também em termos da dificuldade de expressar afetos, é uma característica muito forte em muitos hipertensos. Lipp (1990) verificou que quando confrontados com situações nas quais a assertividade seria, em geral, esperada do interlocutor, as pessoas hipertensas responderam com grandes aumentos na pressão arterial em picos de reatividade cardiovascular.

Aumentos da pressão sistólica foram encontrados inversamente correlacionados com a expressão de alegria e felicidade (James, Yee, Harshfield, Blank \& Pickering, 1986). Foi também demonstrado que dependendo da emoção eliciada pode haver aumentos da pressão arterial sistólica, da diastólica, ou de ambas (Holt-Lunstad, Uchino, Smith, Olsen-Cerny \& Nealey-Moore, 2003; Spielberger, Crane, Kearns, Pellegrin \& Rickman, 1991), porém não se encontraram estudos que averiguassem a magnitude da reatividade cardiovascular, em situações de stress, em função de características psicológicas da pessoa, não estando ainda claro até que ponto as características personológicas contribuem para a magnitude da reatividade cardiovascular em momentos de stress interpessoal. Este trabalho objetivou verificar se a reatividade cardiovascular de 
hipertensos leves é influenciada, em momentos de stress emocional, pelo fato de a pessoa ser alexitímica ou inassertiva.

\section{Método}

\section{Participantes}

Oitenta adultos (28 homens e 52 mulheres) com idade média de 42,1 anos e desvio-padrão de 1,3 anos, que atenderam a anúncios de um estudo que estava sendo realizado pelo Laboratório de Estudos Psicofisiológicos do Stress da Pontifícia Universidade Católica de Campinas sobre possíveis efeitos do stress na pressão arterial de hipertensos. Os participantes foram selecionados dentre mais de 200 que se inscreveram para o estudo. Todos os participantes haviam sido diagnosticados por cardiologistas como sendo hipertensos do Estágio 1 (pressão arterial sistólica entre 140 e 159mmHg e pressão diastólica entre 90 e 99mmHg). Nenhum estava tomando medicação hipotensora na época da avaliação.

\section{Material}

Para coleta de dados psicológicos foram utilizados: termo de consentimento, Questionário de Assertividade, Escala para Diagnóstico de Alexitimia, Inventário de Sintomas do Stress para Adultos (ISSL). Utilizaram-se também fitas contendo quatro cenas que em pesquisas anteriores (Lipp, 1997) foram utilizadas para criarem stress social nos participantes. As fitas utilizadas incluíram duas cenas sobre situações negativas/ conflitivas e duas de conteúdo positivo.

O Questionário de Assertividade utilizado foi elaborado com base nos princípios de Rathus e contém 17 itens aos quais a pessoa responde sim ou não. Sete respostas inassertivas é o estabelecido para determinar se o respondente é inassertivo. Esse questionário já foi utilizado em várias pesquisas (Lipp, 1997). O ISSL (Lipp, 2000) contém três quadros que correspondem às fases do stress. O ISSL possibilita determinar se a pessoa tem stress, em que fase se encontra e se existe prevalência de sintomas somáticos ou cognitivos. A Escala de Alexitimia de Toronto foi traduzida e validada por Yoshida (2003).
A pressão arterial e freqüência cardíaca eram aferidas continuamente através do uso do Monitor de Pressão Arterial Finapress, marca Ohmeda, modelo 2300, Denver, Colorado, que mede a pressão arterial e a freqüência cardíaca continuamente. O Finapress é um equipamento que afere a pressão arterial e a freqüência cardíaca, de modo não invasivo, através de uma pequena cuff' equipada com um fotopletismógrafo, que é colocada ao redor do dedo médio da mão não dominante do participante. O manguito é conectado a uma caixa pequena contendo uma válvula pneumática, ligada a uma fonte de compressão de ar, um transdutor eletropneumático, e componentes eletrônicos para o pletismógrafo. O ponto de compressão do volume sangüíneo é ajustado periodicamente para permitir que a pressão reflita continuamente a pressão intra-arterial. Ao ser conectado ao computador, fornece a listagem das medidas registradas, fornecendo as médias de dez em dez segundos.

\section{Procedimentos}

Alguns dias antes da sessão experimental, as pessoas que se inscreveram para participarem do estudo e que haviam sido diagnosticadas como portadoras de hipertensão arterial do Estágio I receberam explicações detalhadas sobre o procedimento do estudo e assinaram um Termo de Consentimento previamente aprovado pela Comissão de Ética. A seguir, responderam aos testes psicológicos e após a avaliação foi marcada a sessão experimental.

A tarefa experimental utilizada foi participar de duas dramatizações (role play). Cada dramatização era constituída de uma interação social, no laboratório, onde as cenas nas quais as pessoas deviam participar eram apresentadas em fitas de áudio.

O participante era solicitado a interagir com uma experimentadora como se estivesse vivenciando a situação apresentada. Dois tipos de instruções foram dadas; em um momento a pessoa era instruída a controlar suas emoções e a agir o mais polidamente possível, não revelando o que sentia (condição "inibir") e em outro momento a instrução era fornecida para que expressassem os sentimentos sem inibições (condição"expressar"). Cada pessoa participou das duas condições, variando somente a ordem de apresentação da instrução. 
As instruções foram fornecidas de modo contrabalançado, de maneira que 40 dos participantes receberam primeiro a instrução "inibir" e na segunda sessão receberam a instrução "expressar"e os outros 40 receberam as instruções na ordem inversa. O cuidado tomado em balancear as instruções visou evitar o viés da pessoa sempre ouvir uma instrução ou outra em primeiro lugar. A pressão arterial e a freqüência cardíaca foram aferidas continuamente.

Os resultados foram analisados em função de cada momento da sessão experimental, que constituiu de uma linha de base inicial e outra final, duas cenas positivas e duas cenas negativas ou conflitivas, cada uma precedida de um período no qual a pessoa ouvia a descrição da cena a ser respondida, em um total de 10 momentos.

A reatividade cardiovascular foi calculada subtraindo-se a média da pressão arterial sistólica (PAS), diastólica (PAD), pressão arterial média (PAM) e freqüência cardíaca (FC) obtidas na primeira linha de base das aferidas em todos os outros momentos de stress experimental. Desse modo, obteve-se um índice de reatividade das variáveis cardiovasculares estudadas para cada momento do experimento. Inicialmente, a análise dos dados foi realizada a fim de verificar se a ordem das instruções (inibir ou expressar emoções) teve um efeito significativo na reatividade cardiovascular dos participantes. A seguir foi averiguado como a condição de expressar ou não emoções interagia com as características psicológicas para determinar o nível de reatividade cardiovascular em momentos de stress. Todas as análises estabeleceram o valor de $p \leq 0,05$.

\section{Resultados}

Não houve diferença significativa quanto a ouvir primeiro ou depois as instruções para inibir ou para expressar emoções, isto é, a ordem da apresentação não afetou significativamente a pressão arterial média ou a freqüência cardíaca durante o estudo, de modo que essa variável não foi considerada nas demais análises. Análise de variância, levando em consideração todas as variáveis psicológicas e as medidas de reatividade, mostrou que a reatividade da pressão arterial diastólica foi significativamente mais alta em participantes 164 alexitímicos $\left(F_{(1,73)}=5,59 ; p=0,021\right)$ na condição de expressar emoções e que a PAS foi significativamente maior na condição de inibir emoções de acordo com o nível menos elevado de alexitimia do respondente $\left(F_{(1,73)}=4,34 ; p=0,041\right)$, isto é, os alexitímicos exibiam menor reatividade quando solicitados a não expressarem emoções. Com relação à $\mathrm{FC}$, os dados indicaram que a reatividade foi significativamente menor em pessoas em níveis avançados de stress quando elas foram solicitadas a inibirem suas emoções $\left(F_{(1,73)}=4,94\right.$; $p=0,029$ ).

A influência das características psicológicas fica mais clara quando se calculam os coeficientes de correlação de Pearson entre a reatividade cardiovascular em cada um dos momentos do estudo e os escores das variáveis psicológicas (fase de stress, escores de assertividade e alexitimia), respectivamente, para as situações de "expressar"e"inibir emoções". Verificou-se que a reatividade da PAD exibiu uma correlação positiva significativa com níveis de alexitimia $(r=0,278 ; p=0,013)$ e negativa com os de assertividade $(r=-0,228 ; p=0,044)$ durante o período em que os participantes ouviam a descrição da cena positiva, ou seja, enquanto se preparavam para responderem a uma interação considerada desejável do ponto de vista social, como agradecer um favor ou fazer um elogio. A PAD também se relacionou de forma negativa com o nível de stress do respondente enquanto este se preparava para responder à cena negativa $(r=-0,254 ; p=0,024)$, como discordar da pesquisadora ou defender um direito que havia sido negado, na condição de expressar emoções. Os dados indicam que a expectativa de ter que agir de um modo ou de outro influencia de modo significativo a pressão arterial do momento, dependendo do nível de stress e de assertividade e alexitimia da pessoa.

No que se refere à reatividade da PAS, verificou-se coeficientes de correlação positivos com os níveis de alexitimia nos seguintes momentos: ouvir as descrições das cenas positiva $(r=0,282 ; p=0,012)$ e negativa $(r=0,283 ; p=0,011)$, responder durante a cena positiva $(r=0,367 ; p<0,001)$ e negativa $(r=0,228 ; p=0,043)$. As correlações entre a reatividade da PAS com níveis de assertividade apresentaram valor negativo, de modo que quanto mais inassertiva a pessoa era, maior era a reatividade da PAS durante os seguintes momentos; ouvir as descrições das cenas positiva $(r=-0,226 ; p=0,045)$ e negativa $(r=-0,263 ; p=0,019)$ nas quais teria que se 
engajar e também durante o período que estava interagindo com a pesquisadora na cena positiva $(r=-0,288$; $p=0,010)$ e negativa $(r=-0,265 ; p=0,018)$. A PAM se correlacionou significativamente apenas durante o período em que o respondente ouvia a descrição da cena positiva, no que se refere à alexitimia $(r=0,288 ; p=0,010)$ e à assertividade $(r=-0,256 ; p=0,023)$.

Quanto à reatividade da PAS, verificou-se correlação significativa positiva com assertividade e negativa com alexitimia na condição de inibir emoções, isto é, quando a pessoa assertiva era solicitada a inibir suas emoções e a agir de modo como se não estivesse percebendo o aspecto conflitivo da interação social na qual estava engajada, sua PAS aumentava significativamente. Se a pessoa não era alexitímica, a PAS também subia.

No que se refere à reatividade da freqüência cardíaca, somente foram encontradas correlações negativas durante o período de escuta com o nível de stress do respondente $(r=-0,333 ; p=0,003$ para cenas positivas e $r=-0,230 ; p=0,042$ para as negativas).

\section{Discussão}

Os dados obtidos dão apoio à noção de que emoções distintas geram reatividades diferentes. A PAD e a PAS não variaram sempre de modo paralelo; em certos momentos uma aumentava enquanto a outra não sofria mudanças, dependendo da condição experimental em vigor e das características psicológicas do participante. Tipicamente, aumentos significativos da PAD ocorriam na condição em que a pessoa era solicitada a expressar emoções de modo livre, enquanto que a reatividade da PAS era maior na situação em que se solicitava ao participante que não expressasse seus sentimentos durante períodos de stress interpessoal nos quais seus direitos haviam sido violados, ou quando ele tinha que expressar uma opinião que divergia da pesquisadora.

A PAD variou especialmente na condição em que as pessoas eram solicitadas a ouvirem a descrição de cenas positivas e responderem a elas expressando livremente o que sentiam. Quando solicitadas a não demonstrarem emoção em tais momentos, a PAD não mostrou aumentos significativos independentemente dos traços psicológicos.
Os resultados apóiam o proposto por James et al. (1986), Spielberger et al. (1991) e Holt-Lunstad et al. (2003) quanto ao fato de que emoções diferentes geram maior reatividade na PAD ou na PAS, pois revelam a influência dos fatores psicológicos no nível de stress eliciado que se manifestou na forma de efeitos diferenciais na reatividade cardiovascular de hipertensos leves. Tanto a alexitimia como a assertividade tiveram influência significativa em alguns aspectos estudados. Por exemplo, pacientes alexitímicos tiveram maior reatividade da PAD quando levados a expressarem emoções, enquanto esse efeito não se manifestou na PAS. Por outro lado, a inibição dos sentimentos, regulados por instruções específicas quanto a não serem expressos, resultou em aumentos pressóricos significativos na PAS e não na PAD. Níveis de alexitimia se correlacionaram com aumentos significativos da PAD durante o período em que os respondentes se preparavam mentalmente para interagirem em contextos considerados positivos, como agradecer a alguém um favor.

No que tange à variável assertividade, é interessante notar que foi verificada uma associação oposta ao percebido referente à alexitimia, o que era previsto, uma vez que os dois conceitos apresentam dimensões opostas. Isto é, o alexitímico tem dificuldades em lidar com as emoções e o assertivo é o que consegue identificar e expressar o que sente.

Nesse aspecto foi identificado que aumentos significativos na PAD se correlacionaram negativamente com os níveis de assertividade, de modo que quanto menos assertivo era o respondente maior era a reatividade da PAD durante o período em que a pessoa estava ouvindo a descrição das cenas positivas nas quais ela teria que expressar afeto ou agradecimento. Esse dado revela que aspectos da inassertividade, já bastante estudados no público no geral (Ryan, Anas \& Friedman, 2006), têm um impacto que vai além do âmbito psicológico e se expande até aspectos do funcionamento cardiovascular.

Encontrou-se uma correlação negativa entre a reatividade da PAD e níveis elevados de stress quando os respondentes, independentemente de suas características psicológicas, ouviam a descrição das cenas negativas nas quais teriam que defender um direito ou reagirem a uma atitude ofensiva dos outros na condição de expressar. 
Os dados indicam que a expectativa de ter que agir de um modo ou de outro influencia de maneira significativa a pressão arterial diastólica do momento, dependendo do nível de stress, assertividade e alexitimia da pessoa envolvida.

A PAS variou de modo significativo em situações nas quais as emoções deveriam ser reguladas devido às instruções recebidas. Essa supressão de sentimentos teve efeito na PAS dependendo dos traços psicológicos existentes, como a alexitimia e a assertividade. Quando pessoas alexitímicas e assertivas foram solicitadas para inibirem suas emoções enquanto interagiam com a pesquisadora em situações de grande stress, sua PAS mostrou aumentos consideráveis em todos os momentos experimentais, ou seja, quando ouviam a descrição das cenas tanto positivas como negativas e quando respondiam em ambas as situações. Esse dado pode ser compreendido considerando-se que a pessoa assertiva teria, por natureza, a tendência a expressar os sentimentos livremente, enquanto a pessoa alexitímica provavelmente não o faria tão facilmente.

A FC, por outro lado, não foi afetada pelas características psicológicas e sim pelo nível de stress dos participantes na situação de inibição dos sentimentos; quanto maior o nível de stress, menor era a FC. Esse dado talvez possa ser explicado pelo fato de que no início do processo de stress, o organismo produz uma grande quantidade de adrenalina a fim de possibilitar a reação de "luta ou fuga". Com o aumento do nível de stress, há um aumento na produção do cortisol e decréscimo na de adrenalina. Além disto, em uma situação experimental em que a pessoa era solicitada a não reagir e a não mostrar emoções, não havia necessidade do preparo para a reação de "luta ou fuga", o que poderia justificar a pequena reatividade de FC.

\section{Conclusão}

Os dados indicaram que variáveis psicológicas como inassertividade, alexitimia e stress emocional são fatores importantes na determinação da magnitude da reatividade cardiovascular que ocorre em contatos sociais estressantes de pessoas hipertensas leves. As pessoas inassertivas e/ou alexitímicas mostram uma reatividade de PAD maior quando são solicitadas a expressarem seus sentimentos; por outro lado, pessoas com essas características tendem a ter uma maior reatividade de PAS quando levadas a regularem suas emoções inibindo a sua demonstração. Considerando a importância que o controle da reatividade cardiovascular tem para a saúde das pessoas hipertensas, medidas psicológicas capazes de reduzirem a inassertividade e a alexitimia devem ser incluídas em programas de tratamento do hipertenso do mesmo modo que, como sugerem as V Diretrizes de Hipertensão Arterial (2006), o treino psicológico de controle do stress deve ser oferecido aos hipertensos a fim de que eles possam adquirir estratégias de enfrentamento do stress excessivo e possam minimizar seus efeitos negativos.

\section{Referências}

Fritz, H. L., Nagurney, A. J., \& Helgeson, V. S. (2003). Social interactions and cardiovascular reactivity during problem disclosure among friends. Personality and Social Psychololy Bulletin, 29 (6), 713-25.

Holt-Lunstad, J., Uchino, B. N., Smith, T. W., Olsen-Cerny, C., \& Nealey-Moore, J. B. (2003). Social relationships and ambulatory blood pressure structural and qualitative predictors of cardiovascular function during everyday social interactions. Health Psychology, 22 (4), 388-397.

James, G., Yee L., Harshfield, G., Blank, S., \& Pickering, T. (1986). The influence of happiness, anger, and anxiety on blood pressure of borderline hypertensives. Psychosomatic Medicine, 48, 502-508.

Kamarck, T. W., Lovallo, W. R. (2003). Cardiovascular reactivity to psychological challenge: conceptual and measurement considerations. Psychosomatic Medical, 65 (1), 9-21.

Light, K. C., Sherwood, A., \& Turner, J. R. (1992). High cardiovascular reactivity to stress: a predictor of later hypertension development. In J. R. Turner, A. Sherwood \& K. C. Light (Eds.), Individual differences in cardiovascular response in stress (pp.281-293). New York: Plenum.

Lipp, M. E. N., \& Rocha, J. C. (1994). Stress, hipertensão e qualidade de vida. Campinas: Papirus.

Lipp, M. E. N. (1990). Stress e hipertensão arterial essencial. In M. F. Oliveira \& S. Cury (Orgs.), Rumos da psicologia hospitalar em cardiologia. Campinas: Papirus.

Lipp, M. E. N. (1997). Pesquisas sobre hipertensão. Arquivos Brasileiros de Cardiologia, 68 (1), 56- 60.

Lipp, M. E. N. (2000). Inventário de sintomas de stress para adultos. São Paulo: Casa do Psicólogo.

Lipp, M. E. N. (2005). Blood pressure reactivity to social stress in an experimental situation. Revista de Ciências Médicas, 14 (4), 313-394.

Lipp, M. E. N., Justo, A. P., \& Melo Gomes, T. (2006). Cardiovascular reactivity in hypertensives: differential effect of expressing and inhibiting emotions during 
moments of interpersonal stress. The Spanish Journal of Psychology, 9 (2), 154-161.

Loures, D. L., Sant'Anna, I., Baldotto, C. S. R., Sousa, E. B., \& Nóbrega, A. C. L. (2002). Estresse mental e sistema cardiovascular. Arquivos Brasileiros de Cardiologia, 78 (5), 525-530.

Lovallo, W. R., \& Gerin, W. (2003). Psychophysiological reactivity: mechanisms and pathways to cardiovascular disease. Psychosomatic Medicine, 65 (1), 36-45.

Ryan, E. B., Anas, A. P., \& Friedman, D. B. (2006). evaluations of older adult assertiveness in problematic clinical encounters. Journal of Language and Social Psychology, 25 (2), 129-145.

Sandin, B., Chorot, P., Santed, M., \& Jimenez, P. (1996). Dimensiones de alexitimia y estados emocionales de ansiedad, depresion e ira. Psiquis: Revista de Psiquiatria, Psicologia y Psicosomatica, 17 (1), 49-57.

Sandin, B., Santed, M., Chorot, P., \& Valiente, R. (1996). Alexitimia, afecto positivo y negativo, y reactividad al estre: relaciones con sintomatologia somatica y enfermedad. Analisis y Modificacion de Conducta, 22 (84), 435-460.

Schwartz. A. R., Gerin, W., Davidson, K. W., Pickerin, T. G., Brosschot, J. F, Thayer, J. F., Christenfeld, N., \& Liden, W. (2003). Toward a causalmodel of cardiovascular responses tostrss and the development of cardiovascular disease. Psychosomatic Medicine, 65 (1) 22-35.

Sheffield, D., Smith, G., Carrol, D., Shipley, M., \& Marmot, M. (1997). The effects of blood pressure resting level and lability on cardiovascular reactions to laboratory stress. International Journal of Psychophysiology, 27 (2), 79-86.

Sifneos, P. E. (1972). Short term psychotherapy and emotional crisis. Cambridge: Harvard University Press.

Spielberger, C., \& Moscoso, M. (1996). Reacciones emocionales del estres: ansiedad y colera. Avances in Psicologia Clinica Latino-Americana, 14, 59-81.

Spielberger, C., Crane, R. S., Kearns, W. D., Pellegrin, K. L., \& Rickman, R. L. (1991) Anger and anxiety in essential hypertension. In C. Spielberger \& I. G. Sarason. Stress and emotion. New York: Hemisphere Publishing Cor.

Treiber, F. A., Kamarck, T., Scheneiderman, N., Sheffield, D., Kapppuku, G., \& Taylor, T. (2003). Cardiovascular reactivity and development of preclinical and clinical disease states. Psychosomatic Medicine, 65 (1), 46-62.

Yoshida, E. M. P. (2003). Toronto Alexithymia Scale- TAS: precisão e validade da versão em português. Psicologia: Teoria e Prática, 2 (1), 59-74.

Wolpe, J. (1973). The practice o behavior therapy. New York: Pergamon Press.

V Diretrizes Brasileiras de Hipertensão Arterial. (2006). São Paulo: Sociedade Brasileira de Cardiologia.

Recebido em: 22/1/2007

Aprovado em: 13/2/2007 
